Kosinski E. P., Stein N., Malindzak G. S. \& Boone E. (1971) Glucagon and propranolol (inderal) toxicity. New England Journal of Medicine, 285, 1325

Porte D. \& Halter J. B. (1981) The endocrine pancreas and diabetes mellitus. In: Textbook of Endocrinology. Williams R. H. (ed), chapter 15, pp 749. W. B. Saunders, Philadelphia, U.S.A.

Weinstein R. S. (1984) Recognition and management of poisoning with beta-adrenergic blocking $\equiv$ agents. Annals of Emergency Medicine 13, 1123-31

Westlie L., Andersen A., Jervell J., Rasmussen K. \& Storstein O. (1971) Cardiovascular effects of glucagon. Acta Medica Scandinavica 189, 179-84

\title{
Is anaesthesia necessary for reducing shoulder dislocation?
}

Sir

It is our experience that, provided it is performed gently and early enough, manipulation of the dislocated shoulder often does not require any adjunctive analgesia or anaesthesia.

The technique used for reduction is based on the Kocher manoeuvre. The $\stackrel{9}{9}$ patient is forewarned that no sudden movements will be used and that over a $\vec{c}$ period of $4-5 \mathrm{~min}$. the arm will be gently manipulated to allow relaxation of the $\frac{\mathbb{0}}{0}$ contracted muscle (i.e. subscapularis) that is keeping the humeral head in the $\frac{\mathbb{\Phi}}{3}$ dislocated position. No patient refused to undergo this manoeuvre, although $\underset{\Phi}{\mathbb{\Phi}}$ several have expressed initial reservations. The patient is then seated on a pedestad, $\overrightarrow{0}$ with no back support, or on a chair ensuring that he or she does not leag \& backwards. An assistant gently supports the humeral head in the axilla, whie standing behind the patient and on the side opposite to the dislocation. The operator flexes the elbow of the injured arm and applies gentle longitudinal downwards traction on the humeral shaft. As traction is applied, gentle external rotation of the humeral shaft is performed. If successful, the humeral head invariably $\stackrel{\square}{\circ}$ slips back into the glenoid cavity. Often, no definite jerk can be elicited as the shoulder reduces. Patients are invariably grateful once this is achieved.

In a 2-year period from April 11988 to April 1 1990, 44 acute anterior dislocations of the shoulder were treated in the Accident and Emergency Department at Walsall. The ages of the patients ranged from 17 to 90 . Of these, 34 were reduced without any adjunctive anaesthesia or muscle relaxant. Of those reductions that failed with the above procedure, 4 were reduced with the aid of intravenous diazepam and 6 required general anaesthesia. No neurovascular complications

were attributable to the reduction in any instance.
Although not widely accepted or recognized, it must be emphasized that the majority of shoulder dislocations can be reduced without any anaesthesia. This is particularly relevant if the patient is unfit for anaesthesia.

\section{A. BANERJEE}

Accident and Emergency Department

Manor Hospital

West Midlands 